\title{
DISCUSSION
}

\section{Asymptotic behaviour of a granular soil in strain path testing}

\author{
J. CHU and S.-C. R. LO (1994). Géotechnique 44, No. 1, 65-82
}

\section{G. Gudehus, University of Karlsruhe}

Recent theoretical concepts may help to make more use of the findings in the Paper.

The SOM behaviour is easily explained using Kolymbas's (1991) concept of hypoplasticity. For cylindrical compression with constant strain ratios $\dot{\varepsilon}_{1}$ and $\dot{\varepsilon}_{2}=\dot{\varepsilon}_{3}$ the stress rates are calculated as

$$
\begin{aligned}
\dot{\sigma}_{1}= & A\left[L_{1}\left(\sigma_{1} / \sigma_{2} ; \dot{\varepsilon}_{1}, \dot{\varepsilon}_{2}\right)\right. \\
& \left.+N_{1}\left(\sigma_{1} / \sigma_{2}\right) \sqrt{ }\left(\dot{\varepsilon}_{1}^{2}+2 \dot{\varepsilon}_{2}^{2}\right)\right] \\
\dot{\sigma}_{2}= & A\left[L_{2}\left(\sigma_{1} / \sigma_{2} ; \dot{\varepsilon}_{1}, \dot{\varepsilon}_{2}\right)\right. \\
& \left.+N_{2}\left(\sigma_{1} / \sigma_{2}\right) \sqrt{ }\left(\dot{\varepsilon}_{1}^{2}+2 \dot{\varepsilon}_{2}^{2}\right)\right]
\end{aligned}
$$

where $A$ is a scalar factor depending on void ratio $e$ and mean pressure $\sigma_{\mathrm{m}}=\left(\sigma_{1}+2 \sigma_{2}\right) / 3, L_{1}$ and $L_{2}$ are functions of the stress ratio $\sigma_{1} / \sigma_{2}$ and linear functions of $\dot{\varepsilon}_{1}$ and $\dot{\varepsilon}_{2}$ and $N_{1}$ and $N_{2}$ are functions of the stress ratio $\sigma_{1} / \sigma_{2}$. The equations are rate-independent so that the same stress path is obtained when using the ratio $\dot{\varepsilon}_{1} / \dot{\varepsilon}_{2}$ instead of $\dot{\varepsilon}_{1}$ and $\dot{\varepsilon}_{2}$. With constant $\dot{\varepsilon}_{1} / \dot{\varepsilon}_{2}, \dot{\sigma}_{1} / \dot{\sigma}_{2}$ and thus also $\sigma_{1} / \sigma_{2}$ tends towards a constant value. This SOM behaviour is likewise obtained for other homogeneous deformations with constant strain rate ratios and was in fact assumed in the development of hypoplasticity.

A more precise inspection shows, however, that this property cannot strictly hold in general. An asymptotic independence of stress ratios can be expected only for deformations with constant volume, i.e. for so-called critical states. Dilatant or contractant deformations cause changes of $e$ and $\sigma_{m}$ until decay of the soil element or total grain crushing makes an end. Following Kolymbas (1991), these effects of pyknotropy and barotropy, (i.e. density and pressure dependence) can be modelled by a combined extension of hypoplasticity. This can be achieved by means of pyknotropy factors $f_{\mathrm{e}}$ and $f_{\mathrm{d}}$ and a barotropy factor $f_{\mathrm{b}}$ depending on $e$ and $\sigma_{\mathrm{m}}$ (Gudehus, 1994). For cylindrical compression this means, instead of equations (8) and (9)

$$
\begin{aligned}
\dot{\sigma}_{1}= & f_{\mathrm{b}} f_{\mathrm{e}}\left[L_{1}\left(\sigma_{1} / \sigma_{2} ; \dot{\varepsilon}_{1}, \dot{\varepsilon}_{2}\right)\right. \\
& \left.+f_{\mathrm{d}} N_{1}\left(\sigma_{1} / \sigma_{2}\right) \sqrt{ }\left(\dot{\varepsilon}_{1}^{2}+2 \dot{\varepsilon}_{2}^{2}\right)\right]
\end{aligned}
$$

$$
\begin{aligned}
\dot{\sigma}= & f_{\mathrm{b}} f_{\mathrm{e}}\left[L_{2}\left(\sigma_{1} / \sigma_{2} ; \dot{\varepsilon}_{1}, \dot{\varepsilon}_{2}\right)\right. \\
& \left.+f_{\mathrm{d}} N_{2}\left(\sigma_{1} / \sigma_{2}\right) \sqrt{ }\left(\dot{\varepsilon}_{1}^{2}+2 \dot{\varepsilon}_{2}^{2}\right)\right]
\end{aligned}
$$

where $f_{\mathrm{d}}$ and $f_{\mathrm{e}}$ depend on the relative void ratios $e / e_{\mathrm{c}}$ and $e / e_{\mathrm{d}}$, and $f_{\mathrm{b}}$ can be calculated from a given dependence of $e$ against $\sigma_{\mathrm{m}}$. The maximum (or critical) and minimum void ratios $e_{\mathrm{c}}$ and $e_{\mathrm{d}}$ decrease with $\sigma_{\mathrm{m}}$ as $e$ for proportional compression. Similar equations are obtained for other modes of deformation from a general tensorial constitutive equation.

By means of these equations the observations in the Paper can be predicted. Only for deformations with constant volume are the asymptotic stress ratios constant. With other constant strain rate ratios, nearly constant stress ratios are obtained only so long as the changes of void ratio and mean stress are rather small. Equations (10) and (11) can also be used for failure states. It is recommended that this rather vague notion should be replaced by a stringent definition of instability which includes bifurcation and localization (Gudehus, 1994). The influence of pore water and air can be allowed for consistently by the modern theory of mixtures. It is then imperative to apply the symbols and sign conventions of mechanics instead of those of soil mechanics.

\section{Authors' reply}

The term 'asymptotic behaviour' as used in the original sense is only to describe a type of prefailure behaviour (mainly, if not exclusively, hardening) of soils along proportional strain paths. To avoid ambiguity in the definition of a zero strain state, we adopt a linear strain path as defined by a constant strain increment ratio. This type of path is also more general than the proportional strain path, and asymptotic (including SOM) behaviour is still observed. These test results can be used as a benchmark in the development of constitutive models. A good constitutive model, such as the hypoplasticity model proposed by Professor Gudehus, should be able to predict asymptotic behaviour for linear strain path.

In addition to establishing a unique relationship between the asymptotic stress ratio and the 
strain increment ratio for both axisymmetric and three-dimensional loading conditions, the relationship between the limit surface and asymptotic behaviour was also established. This relationship is also consistent with the concept of pre-failure strain softening (Chu, Lo \& Lee, 1992). Furthcrmorc, asymptotic behaviour was also observed in the post-failure region, and a similar equation for predicting the asymptotic stress ratio was also established. However, it is not clear whether or not the model proposed by Professor Gudehus can describe these additional features.

The study presented in the Paper was only for a granular soil at a certain density. The stress ranges used in the study were also mainly limited to pre-grain crushing. We may therefore not be in a position to make definitive statements on the effects of 'pyknotropy' and 'barotropy' on asymptotic behaviour. It is certainly useful and important to project the soil behaviour in a more precise and generalized way using a constitutive model. However, the validation of these predictions need to be verified by more experimental obscrvations.

\section{REFERENCES}

Chu, J., Lo, S.-C. R. \& Lee, I. K. (1992). Strain softening of a granular soil in strain path testing. J. Geotech. Engng Am. Soc. Civ. Engrs 118 No. 2, Feb., 191-208.

Gudchus, G. (1994). A comprehensive constitutive equation for granular materials. Soils \& Fdns Jap. Soc. Soil Mech., to be published.

Kolymbas, D. (1991). An outline of hypoplasticity. Arch. Appl. Mech. 61, 143-151. 